\title{
PCL/CHITOSAN BLENDED NANOFIBROUS TUBES MADE BY DUAL SYRINGE ELECTROSPINNING
}

\author{
Martin Hild ${ }^{1}$, Mohammed Fayez Al Rez ${ }^{2 *}$, Dilbar Aibibu ${ }^{1 *}$, Georgios Toskas ${ }^{1}$, Tong Cheng ${ }^{1}$, Ezzedine Laourine ${ }^{1}$ \\ and Chokri Cherif ${ }^{1}$
}

\author{
${ }^{1}$ Institute of Textile Machinery and High Performance Material Technology, Technische Universität Dresden, Germany \\ ${ }^{2}$ Biomedical Technology Department, Applied Medical Sciences College, King Saud University, Riyadh, Saudi Arabia \\ Corresponding Authors: dilbar.aibibu@tu-dresden.de bme.mfalrez@gmail.com
}

\begin{abstract}
:
3D tubular scaffolds made from Poly-(E-caprolactone) (PCL)/chitosan (CS) nanofibres are very promising candidate as vascular grafts in the field of tissue engineering. In this work, the fabrication of PCL/CS-blended nanofibrous tubes with small diameters by electrospinning from separate $P C L$ and CS solutions is studied. The influence of different CS solutions (CS/polyethylene glycol (PEO)/glacial acetic acid (AcOH), CS/trifluoroacetic acid (TFA), CS/ $A c \mathrm{OH}$ ) on fibre formation and producibility of nanofibrous tubes is investigated. Attenuated total reflection Fourier transform infrared spectroscopy (ATR-FTIR) is used to verify the presence of CS in the blended samples. Tensile testing and pore size measurements are done to underline the good prerequisites of the fabricated blended PCL/ $C S$ nanofibrous tubes as potential scaffolds for vascular grafts. Tubes fabricated from the combination of PCL and CS dissolved in $\mathrm{AcOH}$ possesses properties, which are favourable for future cell culture studies.
\end{abstract}

\section{Keywords:}

Poly-(E-caprolactone), PCL, Chitosan, Electrospinning, Nanofibrous Tubes, Polymer blend

\section{Introduction}

Knitted poly(ethylene terephthalate) (PET, Dacron ${ }^{\mathrm{TM}}$ ) and expanded polytetrafluoroethelene (ePTFE) have been regarded as the standard biomaterials for prosthetic vascular grafts in the cardiovascular bypass procedures. Examined over decades of use, both PET and ePTFE grafts have been shown to perform well at diameters $>6 \mathrm{~mm}$, but neither material has been suitable for small-diameter $(<6 \mathrm{~mm})$ applications because of various limitations, such as thrombogenicity or lack of elasticity $[1,2]$. To overcome this restriction, much effort has been put in the development of scaffolds for vascular tissue engineering, which meet all requirements for an optimal vascular graft (e.g. mechanical support, biocompatibility, cell attachment...) [3].

Electrospinning is a versatile method for spinning polymers to fine fibres. Since they possess high porosities and a large surface to volume ratio, electrospun scaffolds can be designed to mimic the natural extracellular matrix (ECM), thus promoting cell attachment and cell ingrowth [4]. The combination of biodegradable polymers, which exhibit good properties for cell material interactions (e.g. biocompatibility, cell ingrowth and cell attachment) with synthetic polymers, which possess the required mechanical strength is a common attempt in vascular tissue engineering [3].

Of these materials, poly-( $\varepsilon$-caprolactone) (PCL) and chitosan (CS) is a promising combination. PCL is a synthetic biodegradable polymer, which is easily electrospinnable from methanol $(\mathrm{MeOH}) /$ chloroform $\left(\mathrm{CHCl}_{3}\right)$ or tetrafluoroethanol (TFE) and ensures the scaffold good mechanical properties but lacks blood and cellular compatibility because of its hydrophobicity $[5,6]$. CS, a partly deacetylated derivative of chitin, is a linear polysaccharide, which is composed of glucosamine and $N$-acetyl glucosamine units linked by $\beta(1$, 4) glycosidic bonds. CS is a biocompatible, biodegradable, nontoxic material with antimicrobial activity [7]. CS nanofibres can be electrospun pure from different solvent systems like glacial acetic acid $(\mathrm{AcOH}) /$ water $[8,9]$, trifluoroacetic acid (TFA)/dichloromethane $\left(\mathrm{CH}_{2} \mathrm{Cl}_{2}\right)$ [10], or in mixture with poly(ethylene oxide) (PEO) [11]. PCL/CS-blended electrospun tubular scaffolds can be realised by several approaches. In most cases, PCL and CS are either dissolved into one solution and directly electrospun [12-14] or dissolved in separate solutions, which are subsequently mixed and electrospun from one syringe [15-17]. Although the electrospinning setup is kept very simple, this approach has certain limitations regarding the ratio of PCL and CS in one solution. The polyelectrolyte properties of CS limit its solubility in acidic solutions. This limited solubility and the chain entanglement problem in acidic conditions, affect the blend of CS with PCL in the same solvent system [12]. In another approach, PCL and CS solutions are prepared and independently electrospun from two syringes, allowing for the fabrication of material gradients $[18,19]$.

Despite the well-known difficulties to fabricate tubular samples with adequate wall thicknesses and tensile strengths, testing of these properties is avoided in most studies [3]. Also, in all present studies, the result of blended PCL/CS electrospinning is a homogeneous nanofibrous mat on which the PCL and CS fibres are not morphologically distinguishable, which could lead to reduced pore sizes [14]. In this study, the electrospinning 
of PCL and CS from independent spinning solutions with a dual-syringe electrospinning setup is studied. The influence of different CS solutions on the fibre formation, fibre morphology, mechanical strength and realisable wall thickness of the tubular samples is studied. A solvent system, which preserves the native fibre morphology and fibre diameter of each component (PCL and CS) and possesses adequate tensile properties, is presented. Fabricated blended PCL/CS nanofibrous tubes are characterised in terms of their suitability as scaffolds for vascular tissue engineering.

\section{Experimental}

\subsection{Materials}

CS (95/500) was provided by Heppe Medical Chitosan, Germany. PCL (Mw=70,000-90,000 Da) was purchased from Sigma Aldrich, USA. De-ionised water, $\mathrm{AcOH}, \mathrm{PEO}, \mathrm{TFA}$, $\mathrm{CH}_{2} \mathrm{Cl}_{2}, \mathrm{CHCl}_{3}$ and $\mathrm{MeOH}$ were used from Merck, Germany.

\subsection{Preparation of electrospinning solutions}

PCL (10 wt\%) was dissolved in $\mathrm{CHCl}_{3} / \mathrm{MeOH}(6: 1 \mathrm{v} / \mathrm{v})$. CSPEO solution was prepared by mixing stock solutions of $\mathrm{CS}$ (2 wt \% CS in $20 \mathrm{vol} \% \mathrm{AcOH}$ aqueous solution) and PEO (3 wt $\%$ PEO in $0.5 \mathrm{M} \mathrm{AcOH}$ aqueous solution) in the ratio $(6: 4$ $\mathrm{v} / \mathrm{v})$. CS-TFA solution was prepared by dissolving CS (6 wt\%) in TFA $/ \mathrm{CH}_{2} \mathrm{Cl}_{2}(7: 3 \mathrm{v} / \mathrm{v})$. CS-AcOH solution was prepared by dissolving $\mathrm{CS}(6 \mathrm{wt} \%$ ) in $90 \mathrm{vol} \% \mathrm{AcOH}$ aqueous solution. All electrospinning solutions were stirred at room temperature for 24 hours and subsequently filtered through a $0.1 \mathrm{~mm}$ PTFE membrane.

\subsection{Electrospinning setup}

The dual syringe electrospinning setup used for the fabrication of the 3D nanofibrous tubes is shown in Figure 1. The syringes (a) (PCL solution) and (b) (CS solutions) were simultaneously fed by syringe pumps and connected to a high voltage generator. As collector, a rotating metal rod (4 $\mathrm{mm}$ diameter) was used. The rotating speed was set at $500 \mathrm{rpm}$. When fabricating the samples, syringe (a) was always loaded with PCL, whereas syringe (b) was consecutively loaded with the different CS solutions. The fixed electrospinning parameters, as obtained from preliminary experiments, are described in Table 1.

\subsection{Characterisation}

\subsubsection{ATR-FTIR}

Attenuated total reflection Fourier transform infrared spectra (ATR-FTIR) were recorded on a Nicolet 677 (Thermo Scientific, USA) in the range of $650-4000 \mathrm{~cm}^{-1}$ with a resolution of $\mathrm{a} \mathrm{cm}^{-1}$ against air as background media. For each measurement, 64 scans were performed.

\subsubsection{Morphology}

The morphology of the electrospun fibres was investigated using a scanning electron microscope (SEM) XL 30 ESEM (Philips Electron Optics, NL). Free image processing software (image J) was used to measure the fibre diameters. Mean fibre diameter \pm standard deviation (SD) was calculated from 100 individual fibres.

\subsubsection{Mechanical characterisation}

Tensile testing of the electrospun tubular PCL/CS scaffolds was carried out with a standard testing machine (Zwick $\mathrm{GmbH}$, Germany). The loading rate was $10 \mathrm{~mm} / \mathrm{min}$, the load cell was $5 \mathrm{~N}$ with a gauge length of $15 \mathrm{~mm}$. The tubular samples were cut into strips of $25 \times 5 \mathrm{~mm}$, the sample thickness was $0.2-0.3 \mathrm{~mm}$. The nominal strength $(\mathrm{kPa})$ of the sample was calculated by the loading force $(\mathrm{N})$ divided by the nominal crosssection area. Twelve samples of each condition were tested, breaking strength and elongation at break were calculated. All quantitative data are presented as mean \pm SD.

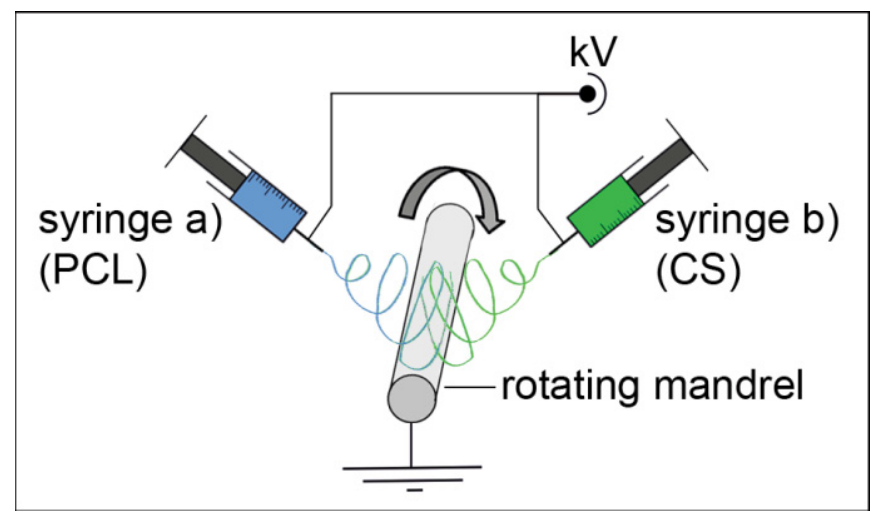

Figure 1 electrospinning setup for the fabrication of PCL/CS-blended nanofibrous tubes

Table 1 Electrospinning parameters

\begin{tabular}{|c|c|c|c|c|c|}
\hline \multicolumn{2}{|c|}{ Syringe/electrospinning solution } & $\begin{array}{c}\text { Working } \\
\text { distance }[\mathrm{cm}]\end{array}$ & $\begin{array}{c}\text { Voltage } \\
\text { [kV] }\end{array}$ & $\begin{array}{c}\text { Feeding rate } \\
{[\mathrm{ml} / \mathrm{h}]}\end{array}$ & $\begin{array}{l}\text { Needle type/ } \\
\text { diameter }\end{array}$ \\
\hline Syringe (a) & PCL & 15 & 20 & 0.2 & G $21 / 0.8 \mathrm{~mm}$ blunt \\
\hline \multirow{3}{*}{ Syringe (b) } & CS-PEO & 10 & 20 & 0.1 & G $23 / 0.4 \mathrm{~mm}$ blunt \\
\hline & CS-TFA & 10 & 20 & 0.1 & G $23 / 0.4 \mathrm{~mm}$ blunt \\
\hline & $\mathrm{CS}-\mathrm{AcOH}$ & 10 & 20 & 0.1 & G $23 / 0.4 \mathrm{~mm}$ blunt \\
\hline
\end{tabular}




\subsubsection{Pore size}

The pore size and pore distribution of the fabricated samples was measured by means of capillary flow porosimetry (pore size meter PSM 165, TOPAS $\mathrm{GmbH}$, Germany) adhering to ASTM E1294-89 and ASTM F316-03, with a capillary constant of 28.6 [20]. As testing fluid, a perfluorocarbon (surface tension $\sigma \mathrm{l}=16 \mathrm{mN} / \mathrm{m}$ ), which secures complete wetting of the scaffolds without swelling, was used. The tubular samples were cut into squares of approximately $12 \times 12 \mathrm{~mm}$. Three samples of each condition were tested to assure better accuracy.

\section{Results and discussion}

\subsection{ATR-FTIR}

The ATR-FTIR spectra of the PCL/CS-blended nanofibrous tubes are shown in Figure 2. All samples show a peak around $1721 \mathrm{~cm}^{-1}$, which is assigned to the $\mathrm{C}=\mathrm{O}$ (carbonyle) stretching of PCL. This peak was comparedly weak for the PCL/CSTFA samples, thus suggesting a lower proportion of PCL. The amide (I) band ( $\mathrm{C}=\mathrm{O}$ stretching) around $1675 \mathrm{~cm}^{-1}$ could only be clearly distinguished at the PCL/CS-TFA samples, since the shoulder of the strong peak around $1721 \mathrm{~cm}^{-1}(\mathrm{C}=\mathrm{O}$ stretching) overlapped with the amide (I) band at the PCL/CS-AcOH and PCL/CS-PEO. The peaks around $1540 \mathrm{~cm}^{-1}$, present at the $\mathrm{PCL} / \mathrm{CS}-\mathrm{AcOH}$ and PCL/CS-TFA samples can be assigned to the amide (II) bands ( $\mathrm{NH}$ bending). The interpretation of the ATR-FTIR spectra suggests that both, PCL and CS in the $\mathrm{PCL} / \mathrm{CS}$-blended nanofibrous samples are present in PCL/CS$\mathrm{AcOH}$ and PCL/CS-TFA samples. Because of the absence of both amide (I) and (II) bands in the PCL/CS-PEO samples, only PCL could be validated.

\subsection{Morphology}

The morphology of the nanofibrous tubes electrospun from different blends of PCL and CS is shown in Figure 3. The observations of the SEM images lead to the assumption that the CS solution strongly affects fibre formation (and thus fibre morphology) in PCL/CS-blended electrospinning. PCL/CSPEO-blended electrospinning (Figure $3 \mathrm{a}, \mathrm{b}$ ) leads neither to a closed nanofibre-mat nor to smooth and uniform fibres. The samples show large beads, which are connected by few nanofibres with diameters in the range of $200-400 \mathrm{~nm}$. Small droplets (assumedly CS) are found between the fibres. Although in pure electrospinning of CS-PEO homogeneous fibre mats can be realised, the presence of PEO in the PCL/CS-PEO electrospinning solutions may prevent both $\mathrm{PCL}$ and $\mathrm{CS}$ from proper fibre formation. The SEM images of CS-PEO match with the ATR-FTIR investigations (see 3.1), proving that there is no significant amount of CS present in the PCL/CS-PEO samples. PCL/CS-TFA samples show a randomly arranged, bead-free structure (Figure $3 \mathrm{c}, \mathrm{d}$ ) with no visible difference between the PCL and CS part. The mean fibre diameter of the PCL/ CS-TFA samples is $299 \pm 62 \mathrm{~nm}$. However, the wall thickness of PCL/CS-TFA electrospun nanofibrous tubes retained very low (max. $0.1 \mathrm{~mm}$ ). This could be due to the interaction of the different electrospinning solutions. Hence, no stable tubular samples could be obtained. PCL/CS-AcOH samples (Figure 3 e, f) exhibit a random fibre alignment. Two different fibre types can be distinguished. Thick fibres (fibre diameter $1204 \pm 221$ $\mathrm{nm}$ ) with an uneven surface are intermingled by a network of very fine fibres (fibre diameter $98 \pm 42 \mathrm{~nm}$ ), which also form few beads. The thick fibres display both similar morphology and fibre diameter ranges as samples electrospun from PCL only, whereas the network of very fine fibres is typical for samples electrospun from CS-AcOH solutions. This conservation of the typical fibre morphologies prompts that the combination of $\mathrm{PCL}$ and $\mathrm{CS}-\mathrm{AcOH}$ seems to cause the least interactions between the different polymer solutions.

Figure 4 shows the fabricated nanofibrous tubes. Tubes fabricated from PCL/CS-TFA solutions did not lead to satisfactory results (Figure 4 a). With PCL/CS-AcOH solutions, stable electrospun tubes with lengths of up to $200 \mathrm{~mm}$ could be achieved (Figure $4 \mathrm{~b}$ ). The wall thickness of PCL/CS-AcOH electrospun nanofibrous tubes could be adjusted to $0.3-0.4$

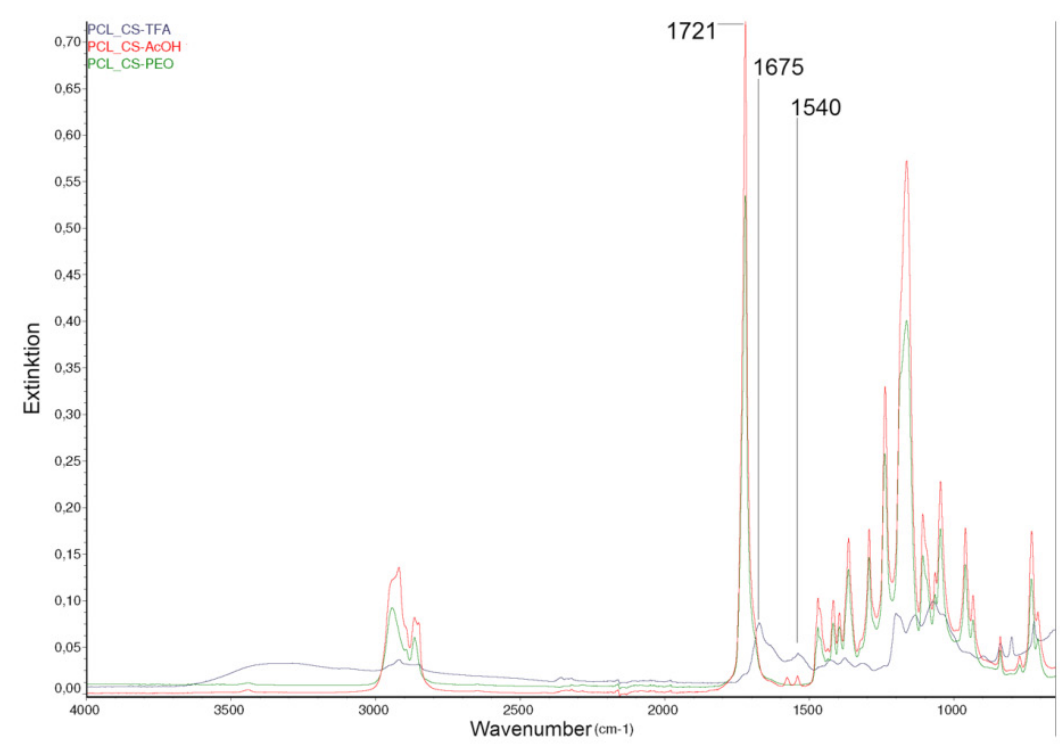

Figure 2 ATR-FTIR spectra of PCL/CS blended nanofibrous tubes (indicated in colour) 

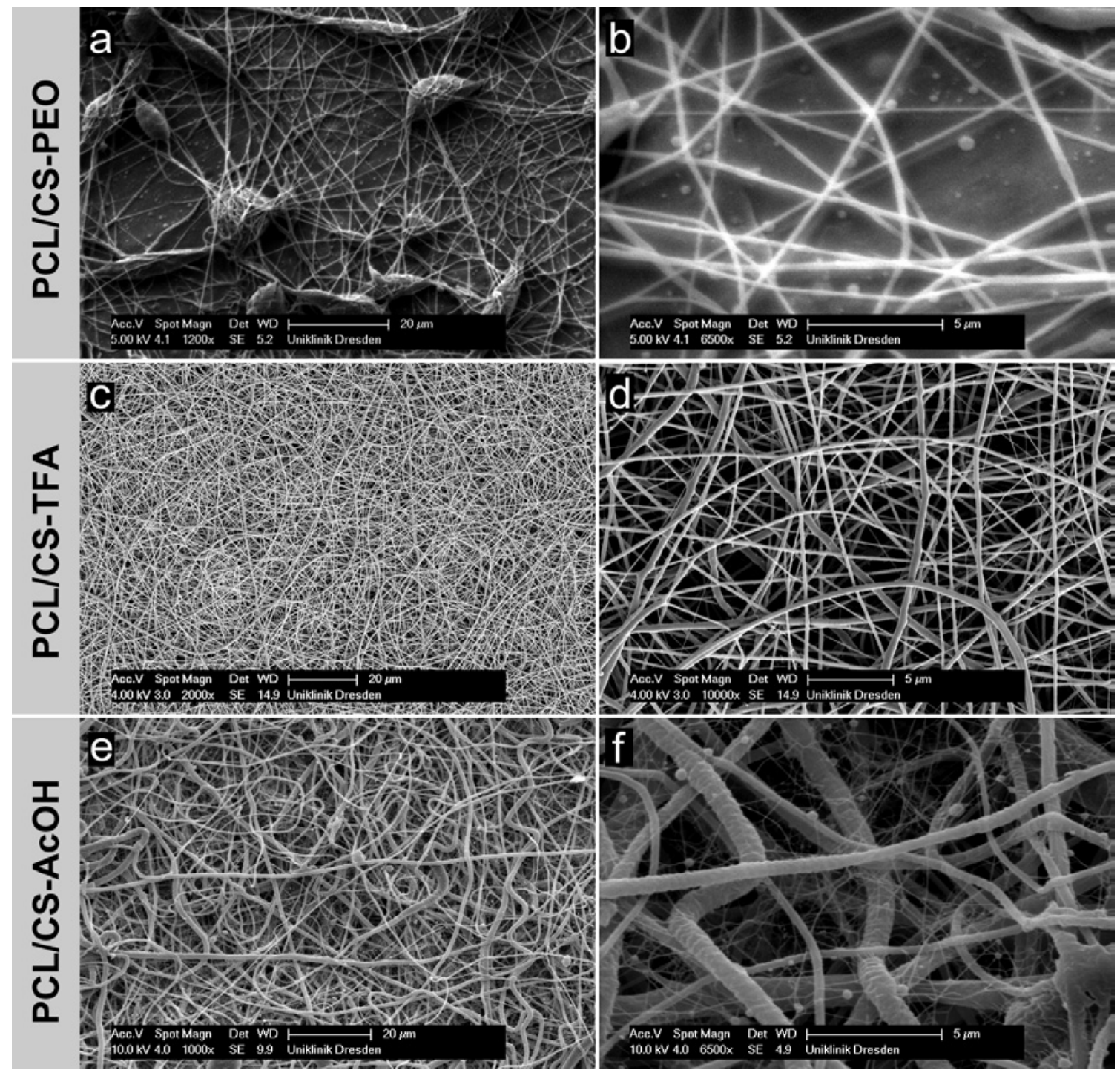

Figure 3: SEM images of the luminal surface of PCL/CS-PEO (a, b), PCL/CS-TFA (c, d), PCL/CS-AcOH (e, f)
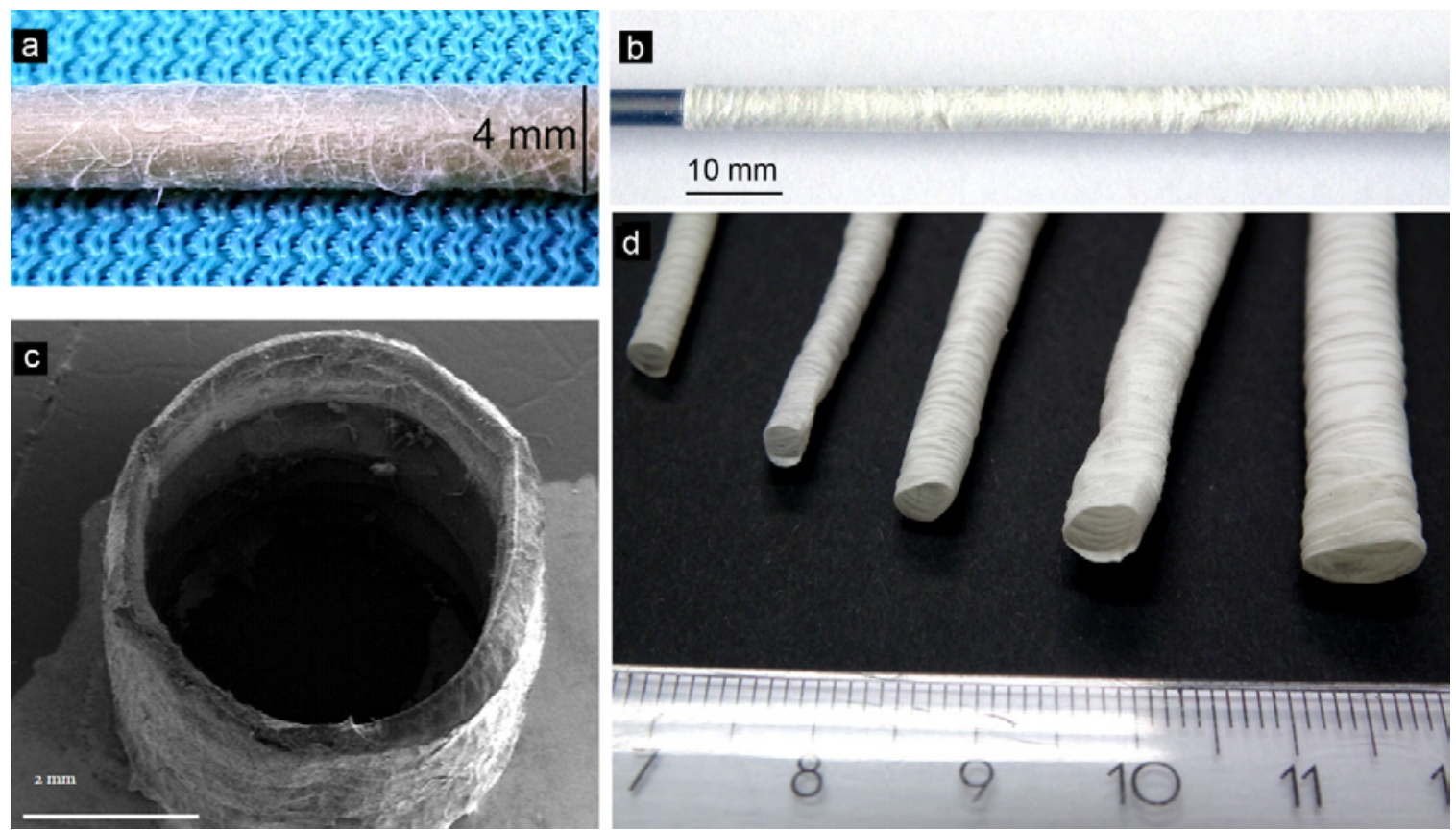

Figure 4: PCL/CS-blended nanofibrous tubes, fabricated from PCL/CS-TFA (a) and PCL/CS-AcOH (b, d) solutions; cross-section of blended nanofibrous PCL/CS-ACOH tube, SEM image (c). 
$\mathrm{mm}$ (Figure $4 \mathrm{c}$ ), which matches the wall thickness of human coronary arteries [21]. The samples fabricated from PCL/CS$\mathrm{AcOH}$ solution led to stable tubes (Figure $4 \mathrm{~d}$ ), which may be used in further applications, for example, vascular tissue engineering.

\subsection{Mechanical characterisation}

Figure 5 shows the tensile strength for the fabricated samples, with a sample made of pure $\mathrm{PCL}$ as reference. The tensile strength of PCL/CS-PEO and PCL/CS-TFA samples could not be measured since the wall thickness of these samples was too small $(<0.1 \mathrm{~mm})$ to obtain stable samples. For the $\mathrm{PCL} / \mathrm{CS}-\mathrm{AcOH}$ samples, a breaking strength of $3010 \pm 540$ $\mathrm{kPa}$ and an elongation at break of $100 \pm 6 \%$ was measured. The reference sample (pure PCL) showed a breaking strength significantly different from PCL/CS-AcOH of $6590 \pm 1080 \mathrm{kPa}$ and an elongation at break of $103 \pm 22 \%$. Typical stress-strain curves are displayed in Figure $5 \mathrm{~b}$. For both PCL and PCL/CS$\mathrm{AcOH}$, the curves show a linear increase until an elongation of approximately $15 \%(\mathrm{PCL})$ and $8 \%(\mathrm{PCL} / \mathrm{CS}-\mathrm{AcOH})$, with initial moduli (measured at $6 \%$ elongation) of $21 \pm 0.8 \mathrm{MPa}(\mathrm{PCL})$ and $6.2 \pm 0.4 \mathrm{MPa}(\mathrm{PCL} / \mathrm{CS}-\mathrm{AcOH})$, respectively. After this linearity, the slope of PCL decreases until fracture, whereas for $\mathrm{PCL} / \mathrm{CS}-\mathrm{AcOH}$ the slope flattens and slightly increases again until fracture. The mechanical characterisation shows that the $\mathrm{PCL}$ present in $\mathrm{PCL} / \mathrm{CS}-\mathrm{AcOH}$ samples accounts for both, their tensile strength and elongation. However, compared to pure PCL, blended PCL/CS nanofibruos tubes possess less tensile strength. The blended $\mathrm{PCL} / \mathrm{CS}-\mathrm{AcOH}$ nanofibrous tubes extend the tensile strength of human coronary arteries, thus matching the tensile requirements as scaffolds for vascular tissue engineering [21]. Also, the elongation of the nanofibrous $\mathrm{PCL} / \mathrm{CS}-\mathrm{AcOH}$ tubes lies in the same range as human coronary arteries [22].

\subsection{Pore size}

Figure 6 shows the pore size distribution of PCL and PCL/CS$\mathrm{AcOH}$ samples. PCL/CS-PEO and PCL/CS-TFA possessed too low wall thicknesses for undamaged measurements. Mean pore sizes were $0.9 \pm 0.4 \mu \mathrm{m}(\mathrm{PCL})$ and $1.1 \pm 0.8 \mu \mathrm{m}$ (PCL/ $\mathrm{CS}-\mathrm{AcOH})$, respectively. Although no significant difference in the mean pore sizes could be determined $(p=0.07)$, the curves of the pore size distribution show that the PCL samples have a peak at approximately $0.9 \mu \mathrm{m}$ and a rather narrow distribution of pore size, whereas the PCL/CS-AcOH samples show a wider pore size distribution with a peak at approximately $1.1 \mu \mathrm{m}$, which is less distinct. The pore size measurements revealed that the blended PCL/CS nanofibrous tubes possess interconnected, microscaled porous structures, thus showing favourable preconditions as scaffolds for vascular tissue engineering [23].
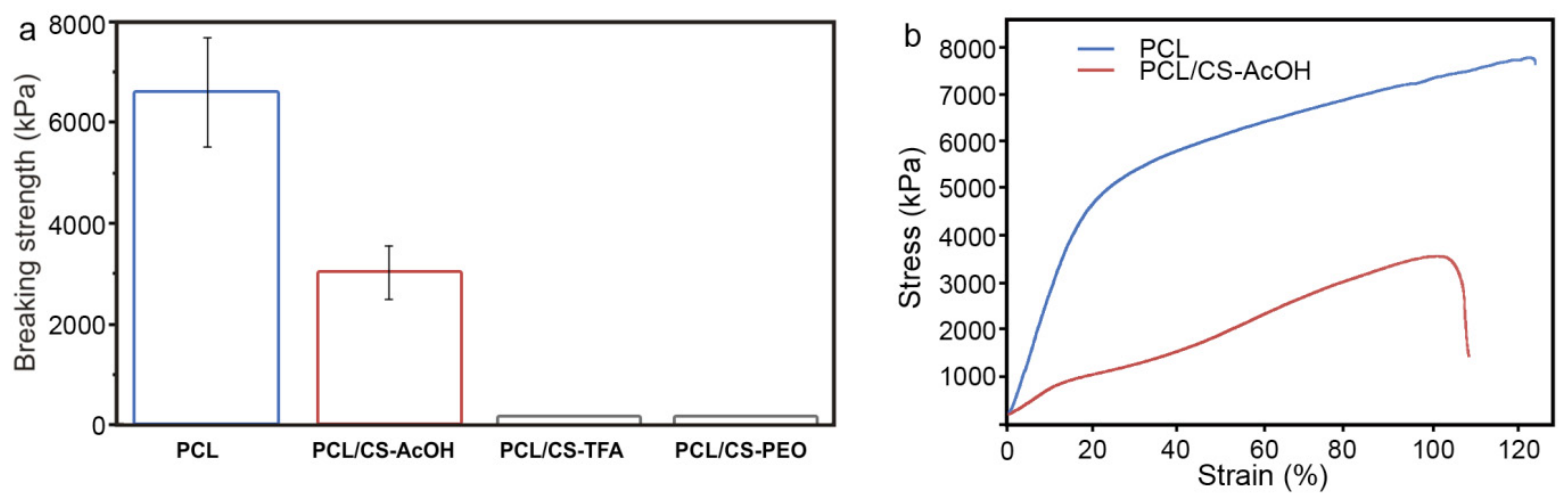

Figure 5: Tensile strength of nanofibrous tubes

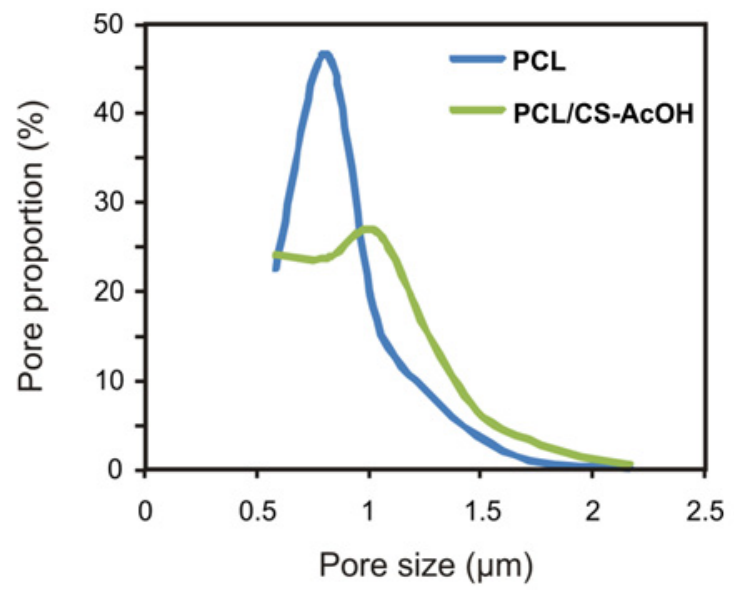

Figure 6: Pore size distribution of PCL and PCL/CS-AcOH nanofibrous tubes 


\section{Conclusions}

This basic research led to mechanically stable tubes made of blended PCL/CS nanofibres. Tubular samples with wall thicknesses $0.3-0.4 \mathrm{~mm}$ and mechanical properties matching native blood vessels [3] could be successfully fabricated, thus proving that mechanically stable grafts may be fabricated by $\mathrm{PCL} / \mathrm{CS}$ tubular electrospinning. The combination of PCL and $\mathrm{CS}$ dissolved in $\mathrm{AcOH}$ proved to be the most suitable solution for the electrospinning of blended PCL/CS nanofibrous tubes. The native fibre morphology of electrospun PCL and CS fibres could be preserved, leading to a hierarchical structure (thick PCL fibres intermingled by a very fine CS-fibre network). By the addition of CS, the mean pore size of the electrospun samples was increased. This could be valuable in cell culture studies since it could be advantageous for cellular ingrowth and enable the selective cell attachment on the CS nanofibres. The performance of these novel blended PCL/CS tubes as scaffolds for vascular tissue engineering will be proved in cell culture experiments. Also, the PCL/CS tubes could be subsequently functionalised for improved cell-material interactions.

\section{ACKNOWLEDGEMENTS}

This work was supported by National Science, Technology and Innovation Plan (NSTIP) strategic technologies program number (09-BIO938-02) in the Kingdom of Saudi Arabia.

\section{References}

[1] Sales KM, Salacinski HJ, Alobaid N, Mikhail M, Balakrishnan $V$ and Am Seifalian. Advancing vascular tissue engineering: the role of stem cell technology. TRENDS IN BIOTECHNOLOGY 2005; 23: 461-467.

[2] Torikai K, Ichikawa H, Hirakawa K, et al. A self-renewing, tissue-engineered vascular graft for arterial reconstruction. JOURNAL OF THORACIC AND CARDIOVASCULAR SURGERY 2008; 136: 37-U56.

[3] Hasan A, Memic A, Annabi $N$, et al. Electrospun scaffolds for tissue engineering of vascular grafts. ACTA BIOMATERIALIA 2014; 10: 11-25.

[4] Li WJ, Laurencin CT, Caterson EJ, Tuan RS and Ko FK. Electrospun nanofibrous structure: A novel scaffold for tissue engineering. JOURNAL OF BIOMEDICAL MATERIALS RESEARCH 2002; 60: 613-621.

[5] Yoshimoto $H$, Shin YM, Terai $H$ and Vacanti JP. $A$ biodegradable nanofiber scaffold by electrospinning and its potential for bone tissue engineering. BIOMATERIALS 2003; 24: 2077-2082.

[6] Shin M, Yoshimoto $H$ and Vacanti JP. In vivo bone tissue engineering using mesenchymal stem cells on a novel electrospun nanofibrous scaffold. TISSUE ENGINEERING 2004; 10: 33-41.

[7] Pillai, C. K. S., Paul W and Sharma CP. Chitin and chitosan polymers: Chemistry, solubility and fiber formation. PROGRESS IN POLYMER SCIENCE 2009; 34: 641-678.

[8] Geng $X Y$, Kwon $\mathrm{OH}$ and Jang JH. Electrospinning of chitosan dissolved in concentrated acetic acid solution. BIOMATERIALS 2005; 26: 5427-5432.

[9] Cheng T, Hund R, Aibibu D, Horakova J and Cherif C. Pure chitosan and chitosan/chitosan lactate blended nanofibres made by single step electrospinning. AUTEX RESEARCH JOURNAL 2013; 13: 128-133.

[10] Ohkawa K, Di Cha, Kim H, Nishida A and Yamamoto $H$. Electrospinning of chitosan. MACROMOLECULAR RAPID COMMUNICATIONS 2004; 25: 1600-1605.

[11] Spasova M, Manolova N, Paneva $D$ and Rashkov I. Preparation of chitosan-containing nanofibres by electrospinning of chitosan/poly(ethylene oxide) blend solutions. E-POLYMERS 2004.

[12] Shalumon KT, Anulekha KH, Girish CM, Prasanth $R$, Nair SV and Jayakumar R. Single step electrospinning of chitosan/poly(caprolactone) nanofibers using formic acid/ acetone solvent mixture. CARBOHYDRATE POLYMERS 2010; 80: 413-419.

[13] Van der Schueren, Lien, Steyaert I, Schoenmaker B de and Clerck K de. Polycaprolactone/chitosan blend nanofibres electrospun from an acetic acid/formic acid solvent system. CARBOHYDRATE POLYMERS 2012; 88: 1221-1226.

[14] Chen H, Huang J, Yu J, Liu S and Gu P. Electrospun chitosan-graft-poly (epsilon-caprolactone)/poly (epsiloncaprolactone) cationic nanofibrous mats as potential scaffolds for skin tissue engineering. INTERNATIONAL JOURNAL OF BIOLOGICAL MACROMOLECULES 2011; 48: 13-19.

[15] Cooper A, Jana S, Bhattarai $N$ and Zhang M. Aligned chitosan-based nanofibers for enhanced myogenesis. JOURNAL OF MATERIALS CHEMISTRY 2010; 20: 89048911.

[16] Roozbahani $F$, Sultana N, Ismail AF and Nouparvar $H$. Effects of Chitosan Alkali Pretreatment on the Preparation of Electrospun PCL/Chitosan Blend Nanofibrous Scaffolds for Tissue Engineering Application. JOURNAL OF NANOMATERIALS 2013.

[17] Jana $S$ and Zhang M. Fabrication of 3D aligned nanofibrous tubes by direct electrospinning. JOURNAL OFMATERIALS CHEMISTRY B 2013; 1: 2575-2581.

[18] Du F, Wang H, Zhao W, et al. Gradient nanofibrous chitosan/ poly epsilon-caprolactone scaffolds as extracellular microenvironments for vascular tissue engineering. BIOMATERIALS 2012; 33: 762-770.

[19] Yao Y, Wang J, Cui Y, et al. Effect of sustained heparin release from $\mathrm{PCL} /$ chitosan hybrid small-diameter vascular grafts on anti-thrombogenic property and endothelialization. ACTA BIOMATERIALIA 2014; 10: 2739-2749.

[20] Huang $C$, Chen $R$, Ke $Q$, Morsi $Y$, Zhang $K$ and Mo $X$. Electrospun collagen-chitosan-TPU nanofibrous scaffolds for tissue engineered tubular grafts. COLLOIDS AND SURFACES B-BIOINTERFACES 2011; 82: 307-315.

[21] Holzapfel GA, Sommer G, Gasser CT and Regitnig P. Determination of layer-specific mechanical properties of human coronary arteries with nonatherosclerotic intimal thickening and related constitutive modeling. AMERICAN JOURNAL OF PHYSIOLOGY-HEART AND CIRCULATORY PHYSIOLOGY 2005; 289: H2048-H2058.

[22] Valenta J. Clinical Aspects of biomedicine. In: Valenta J (ed.) Biomechanics. 2 ed. Amsterdam, New York: Elsevier, (C)1993, pp. 142-179.

[23] Murugan R and Ramakrishna S. Nano-featured scaffolds for tissue engineering: A review of spinning methodologies. TISSUE ENGINEERING 2006; 12: 435-447. 